International Journal of Pure and Applied Mathematics

Volume $116 \quad$ No. $1 \quad 2017,75-90$

ISSN: 1311-8080 (printed version); ISSN: 1314-3395 (on-line version)

url: http://www.ijpam.eu

doi: 10.12732 /ijpam.v116i1.7

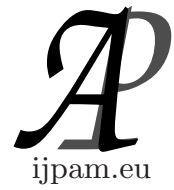

\title{
DERIVATIONS OF FUZZY IMPLICATIVE IDEALS
}

\author{
T.F. Alsolami ${ }^{1}$, S.A. Bashammakh ${ }^{2}$ \\ ${ }^{1}$ Department of Mathematics \\ Faculty of Science \\ Um-AlQura University \\ Makkah, SAUDI ARABIA \\ ${ }^{2}$ Department of Mathematics \\ Faculty of Science \\ King Abdulaziz University \\ Jeddah, SAUDI ARABIA
}

\begin{abstract}
The present paper introduce the notion of (left $\backslash$ right) derivation of $\mathbb{B} \mathbb{C} \mathbb{I}$-algebra $X$ on fuzzy $\mathbb{B C I}$ - ideals and fuzzy implicative ideals and prove various properties. Moreover, we present the relationship between derivation fuzzy implicative ideals and fuzzy commutative and positive implicative ideals through proved theorem.
\end{abstract}

AMS Subject Classification: 06F35, 13N15, 13A15

Key Words: derivations, fuzzy ideal, derivation fuzzy $\mathbb{B} \mathbb{C} I$-ideal, derivation fuzzy implicative ideal, derivation fuzzy commutative ideal, derivation fuzzy positive implicative ideal

\section{Introduction}

In 1965, Zadeh [19] defined the fuzzy set and then many authors investigated this concept and applied it on different branches of mathematics.After that 1966, a new structure in mathematics was obtained by Imai and Iseki called

Received: $\quad$ February 6, 2017

Revised: $\quad$ May 1, 2017

Published: August 29, 2017

(c) 2017 Academic Publications, Ltd. url: www.acadpubl.eu

$\S_{\text {Correspondence author }}$ 
$\mathbb{B} \mathbb{C}$-algbera see $[5,7,14]$.Then Many studies appeared on $\mathbb{B} \mathbb{C} \mathbb{K} \backslash \mathbb{B} \mathbb{C I}$-algbera and its properties.In $1991 \mathrm{Xi}$ was the one who presented the idea of applying fuzzy set to $\mathbb{B C} \mathbb{K}$-algebra[18], consequently many researchers was interested in this notion and introduced more searches about fuzzy $\mathbb{B} \mathbb{C} \mathbb{K}$-algebra and fuzzy ideals[13]. Fuzzy commutative ideals and fuzzy positive implicative ideals studied by Jun and Roh and Jun et al respectively [9], [11].Furthermore, fuzzy implicative ideals and the relation between fuzzy implicative ideals and both commutative and positive implicative ideals was introduced by Meng et al in[15]. The concept of derivation which applied firstly on rings and near rings presented by so many authors in $[3,4,12,17]$.Subsequently, Jun and Xin in [10] proposed derivation on $\mathbb{B} \mathbb{C} \mathbb{I}$-algebra and many results were obtained.Recently, Aboujabal and Alshehri in [1] discussed the derivation on $\mathbb{B} \mathbb{C} \mathbb{K}$-algebra. Moreover, in 2007 [2] they defined a left derivation in $\mathbb{B} \mathbb{C} I$-algebras and investigated a regular left derivation.

At this paper our main goal is to apply the concept of derivation to fuzzy implicative ideals and find various results, studying the relationship between derivation fuzzy implicative ideals and derivation fuzzy commutative and positive implicative ideals and more results are introduced.

\section{Preliminaries}

A $\mathbb{B C I}$-algebra is an algebra of type $(2,0)$ if it satisfies the following axioms for all $x, y, z \in X$ :

(I) $((x * y) *(x * z)) *(z * y)=0$

(II) $(x *(x * y)) * y=0$

(III) $x * x=0$

(IV) $x * y=0$ and $y * x=0$ imply $x=y$

A $\mathbb{B C I}$ is a $\mathbb{B C} \mathbb{K}$-algebra if it satisfies:

(IIV) $0 * x=0$

$\mathbb{B C} \mathbb{K}$ is a generalization of $\mathbb{B} \mathbb{C} \mathbb{I}$-algebra $X$ and a partially ordered relation $" \leq "$ is defined by:

$$
x \leq y \text { if and only if } x * y=0 \text { for all } x, y \in X .
$$

Any $\mathbb{B C I}$-algebra $X$ satisfying the following properties: 
(2.1) $(x * y) * z=(x * z) * y$

(2.2) $x * 0=x$

(2.3) $0 *(x * y)=(0 * x) *(0 * y)$

(2.4) $0 *(0 *(x * y))=0 *(y * x)$

$(2.5)(x * z) *(y * z) \leq x * y$

(2.6) $x * y=0$ implies $x * z \leq y * z$ and $z * y \leq z * x$

For more details of $\mathbb{B} \mathbb{C} \mathbb{K} \backslash \mathbb{B} \mathbb{C I}$-algebras we refer the reader to Iseki and Tanaka $[5,7]$.

A nonempty subset $I$ of a $\mathbb{B} \mathbb{C I}$-algebra $X$ is called an ideal of $\mathrm{X}$ if $\left(I_{1}\right): 0 \in$ $I ;\left(I_{2}\right): x * y \in I$ and $y \in I$ imply $x \in I$. A nonempty subset $I$ of a $\mathbb{B} \mathbb{C I}$-algebra $X$ is called a commutative ideal if it satisfies $\left(I_{1}\right)$ and $\left(I_{3}\right):(x * y) * z \in I$ and $z \in I$ imply $x *(y *(y * x)) \in I$;positive implicative ideal if it satisfies $\left(I_{1}\right)$ and $\left(I_{4}\right):(x * y) * z \in I$ and $y * z \in I$ imply $x * z \in I$; an implicative ideal if it satisfies $\left(I_{1}\right)$ and $\left(I_{5}\right):(x *(y * x)) * z \in I$ and $z \in I$ imply $x \in I$. [6].

A non-empty subset $S$ of a $\mathbb{B} \mathbb{C} \mathbb{K}$-algebra $X$ is called $\mathbb{B} \mathbb{C} \mathbb{K}$-sub-algebra of $X$ if: $x * y \in S$ for all $x, y \in S[7]$

Definition 2.1. (Zadeh [19]) Let $S$ be a set. A fuzzy set in $S$ is a function $\mu: S \longrightarrow[0,1]$.

Definition 2.2. [9] Let $\mu$ be a fuzzy set in a set $S$. For $t \in[0,1]$, the set $\mu_{t}=\{s \in S \mid \mu(s) \geq t\}$ is called a level subset of $\mu$.

Proposition 2.3. [19] $\mu$ and $\nu$ be two fuzzy subsets in a $\mathbb{B} \mathbb{C} \mathbb{K}$-algebra $X$. If $\mu \leq \nu$ Then $\mu(x) \leq \nu(x)$ for any $x \in X$.

Definition 2.4. [18] let $X$ be a $\mathbb{B} \mathbb{C} \mathbb{K}$-algebra. A fuzzy subset $\mu$ in $X$ is said to be a fuzzy sub-algebra of $X$ if:

$$
\mu(x * y) \geq \min \{\mu(x), \mu(y)\} \text { for all } x, y \text { in } X .
$$

Definition 2.5. (Xi [18]) Let $X$ be a $\mathbb{B} \mathbb{C I}$-algebra. A fuzzy subset $\mu$ in $X$ is said to be a fuzzy ideal of $X$ if it satisfies:

$\left(\mathrm{F}_{1}\right) \mu(0) \geq \mu(x)$

$\left(\mathrm{F}_{2}\right) \mu(x) \geq \min \{\mu(x * y), \mu(y)\}$ for all $x, y \in X$

Proposition 2.6. Every fuzzy ideal $\mu$ of a $\mathbb{B} \mathbb{C} \mathbb{I}$-algebra $X$ is order reversing, see [11]. 
Proposition 2.7. [9] Let $\mu$ be a fuzzy ideal of a $\mathbb{B} \mathbb{C} \mathbb{I}$-algebra $X$. Then $x * y \leq z$ implies $\mu(x) \geq \min \{\mu(y), \mu(z)\}$ for all $x ; y ; z \in X$.

Definition 2.8. [8] A fuzzy ideal $\mu$ in a $\mathbb{B} \mathbb{C} \mathbb{I}$-algebra $X$ is said to be closed if for all $x \in X$ :

$$
\mu(0 * x) \geq \mu(x)
$$

\section{Derivations of Fuzzy Implicative Ideals}

In this section, we are going to apply the notion of derivations on fuzzy $\mathbb{B C I}$ ideals and fuzzy implicative ideals of $\mathbb{B} \mathbb{C} \mathbb{I}$-algebra $\mathrm{X}$, But first of all we will introduce the definition of derivation on $\mathbb{B} \mathbb{C} I$-algebra, let $(X, *, 0)$ be a $\mathbb{B} \mathbb{C} I-$ algebra. A map $d: X \longrightarrow X$ is called a left-right derivation (briefly $(l, r)$ derivation) of $X$ if:

$$
d(x * y)=(d(x) * y) \Lambda(x * d(y)), \text { for all } x, y \in X
$$

Similarly, A map $d: X \longrightarrow X$ is called a right-left derivation (briefly $(r, l)$ derivation) of $X$ if:

$$
d(x * y)=(x * d(y)) \Lambda(d(x) * y), \text { for all } x, y \in X
$$

A map $d: X \longrightarrow X$ is called a derivation of $X$ if $d$ is both a $(l, r)$-derivation and a $(r, l)$-derivation of $X$. For more info about derivation on $\mathbb{B} \mathbb{C I}$-algebra see [10].

Definition 3.1. A self map $d$ of $\mathbb{B C} \mathbb{C I}$-algebra is called regular if $d(0)=0$, see $[10]$.

Corollary 3.2. Derivation on $\mathbb{B} \mathbb{C} \mathbb{K}$-algebra is regular, see [1]

Proposition 3.3. Let $d$ be a regular derivation of $\mathbb{B} \mathbb{C I}$-algebra $X$.Then the following are hold for all $x, y \in X$ (see [10]):

(i) $d(x) \leq x$

(ii) $d(x) * y \leq x * d(y)$

(iii) $d(x * y)=d(x) * y \leq d(x) * d(y)$

(iv) $d^{-1}(0)=\{x \in X \mid d(x)=0\}$ is a sub-algebra of $X$ and $d^{-1}(0) \subset X_{+}$ 
Next definitions, we are going to define derivation on fuzzy $\mathbb{B} \mathbb{C I}$-ideal of $\mathbb{B} \mathbb{C} \mathbb{I}$-algebra $X$, but firstly Lets define the derivation concept on $\mathbb{B} \mathbb{C I}$-ideal, $(X, *, 0)$ be a $\mathbb{B} \mathbb{C I}$-algebra, $d: X \longrightarrow X$ be a self map. A non-empty subset $A$ of $\mathbb{B C I}$-algebra $X$ and $x, y, z \in X$ is called left derivation $\mathbb{B} \mathbb{C I}$-ideal of $X$ if it satisfies: $\left(\mathrm{D}_{1}\right) 0 \in A$ and $\left(\mathrm{LD}_{2}\right) d(x) * y \in A$ and $d(y) \in A$ imply $d(x) \in A$;right derivation $\mathbb{B C} \mathbb{C}$-ideal if it satisfies $\left(\mathrm{D}_{1}\right)$ and $\left(\mathrm{RD}_{2}\right) x * d(y) \in A$ and $d(y) \in A$ imply $d(x) \in A$; and called derivation $\mathbb{B} \mathbb{C I}$-ideal if it satisfies $\left(\mathrm{D}_{1}\right)$ and $\left(\mathrm{D}_{2}\right)$ $d(x * y) \in A$ and $d(y) \in A$ imply $d(x) \in A$.

Definition 3.4. Derivation $d: X \longrightarrow X$, fuzzy subset $\mu$ of $\mathbb{B} \mathbb{C I}$-algebra $X$ and a non-empty subset $A$ for all $x, y, z \in X$ is called left derivation fuzzy $\mathbb{B} \mathbb{C} I$-ideal of $X$ if it satisfies: $\left(\mathrm{LDF}_{1}\right) \mu(0) \geq \mu(x)$ and $\left(\mathrm{LDF}_{2}\right) \mu(d(x)) \geq$ $\min \{\mu(d(x) * y), \mu(d(y))\}$; right derivation fuzzy $\mathbb{B} \mathbb{C I}$-ideal of $X$ if it satisfies: $\left(\mathrm{RDF}_{1}\right) \mu(0) \geq \mu(x)$ and $\left(\mathrm{RDF}_{2}\right) \mu(d(x)) \geq \min \{\mu(x * d(y)), \mu(d(y))\}$ and derivation fuzzy $\mathbb{B} \mathbb{C I}$-ideal of $X$ if it satisfies: $\left(\mathrm{DF}_{1}\right) \mu(0) \geq \mu(d(x))$ and $\left(\mathrm{DF}_{2}\right)$ $\mu(d(x)) \geq \min \{\mu(d(x * y)), \mu(d(y))\}$.

Proposition 3.5. Every derivation fuzzy ideal $\mu$ of $X$ is order reversing. $X$.

Proof. Let $d(x) \leq d(y)$, since $\mu$ is derivation fuzzy $\mathbb{B} \mathbb{C I}$-ideal on $\mathbb{B} \mathbb{C} \mathbb{I}$-algebra

By $\left(\mathrm{DF}_{2}\right)$ we have: $\mu(d(x)) \geq \min \{\mu(d(x * y)), \mu(d(y))\}$. Since $d(x) \leq d(y)$ then $d(x) * d(y)=0$. By Proposition $3.3 d(x) * d(y) \geq d(x) * y=d(x * y)$. Hence, $\mu(d(x * y)) \geq \mu(d(x) * d(y))$.

We have:

$$
\begin{aligned}
\mu(d(x)) & \geq \min \{\mu(d(x * y)), \mu(d(y))\} \\
& \geq \min \{\mu(d(x) * d(y)), \mu(d(y))\} \\
& =\min \{\mu(0), \mu(d(y))\} \\
& =\mu(d(y)) .
\end{aligned}
$$

Using $\left(\mathrm{DF}_{1}\right)$, we receive $\mu(d(x)) \geq \mu(d(y))$.

Proposition 3.6. A fuzzy subset $\mu$ satisfying $\left(D F_{1}\right)$ in $\mathbb{B} \mathbb{C I}$-algebra $X$ is a derivation fuzzy $\mathbb{B} \mathbb{C I}$-ideal if and only if for all $x, y, z \in X: d(x * y) \leq d(z)$ implies $\mu(d(x)) \geq \min \{\mu(d(y)), \mu(d(z))\}$

Proof. " $\Rightarrow "$ : Since $\mu$ is derivation fuzzy $\mathbb{B} \mathbb{C I}$-ideal and $d(x * y) * d(z)=0$ implies $d(x * y) \leq d(z)$ by theorem 3.5 we can say $\mu(d(z)) \leq \mu(d(x * y))$ and by $\left(\mathrm{DF}_{2}\right)$ we have $\mu(d(x)) \geq \min \{\mu(d(x * y)), \mu(d(y))\} \geq \min \{\mu(d(z)), \mu(d(y))\}$ so we get: $\mu(d(x)) \geq \min \{\mu(d(z)), \mu(d(y))\}$. 
$" \Leftarrow ": d(x * y) * d(z)=0$ implies $\mu(d(x)) \geq \min \{\mu(d(y)), \mu(d(z))\}$ and $\left(\mathrm{DF}_{1}\right)$ is given, hence left to prove $\left(\mathrm{DF}_{2}\right)$.

Since, $d(x * y) * d(z)=0$ then $d(x * y) \leq d(z)$. We have $\mu(d(x)) \geq$ $\min \{\mu(d(y)), \mu(d(z))\} \geq \min \{\mu(d(y)), \mu(d(x * y))\}$ and the proof is compete.

Definition 3.7. Let $X$ be a $\mathbb{B} \mathbb{C} \mathbb{I}$-algebra. A fuzzy subset $\mu$ in $X$ is said to be a (left/right) derivation fuzzy sub-algebra if for all $x, y$ in $X$ :

$$
\mu(d(x * y)) \geq \min \{\mu(d(x)), \mu(d(y))\} .
$$

Moreover, $\mu$ called (left/right) derivation closed fuzzy $\mathbb{B} \mathbb{C I}$-ideal if $\mu(d(0 *$ $x)) \geq \mu(d(x))$.

Theorem 3.8. Any derivation fuzzy ideal $\mu$ of $X$ must be a derivation fuzzy sub-algebra of $X$.

Proof. Since $d(x * y) \leq d(x)$ it follows from 3.5 that $\mu(d(x)) \leq \mu(d(x * y))$ so by derivation fuzzy ideal properties we have:

$$
\begin{aligned}
\mu(d(x * y)) & \geq \mu(d(x)) \geq \min \{\mu(d(x * y)), \mu(d(y))\} \\
& \geq \min \{\mu d(x), \mu d(y)\} .
\end{aligned}
$$

This shows that $\mu$ is a derivation fuzzy sub-algebra of $X$.

Theorem 3.9. A derivation fuzzy sub-algebra $\mu$ of $X$ is a derivation fuzzy ideal of $X$ if and only if, for all $x, y, z \in X$, the inequality $d(x * y) \leq d(z)$ in $X$ implies that $\mu(d(x)) \geq \min \{\mu(d(y)), \mu(d(z))\}$

Proof. " $\Rightarrow$ ": it follows from derivation fuzzy ideal properties.

$" \Leftarrow "$ : Suppose that $\mu$ is derivation fuzzy sub-algebra of $X$, and satisfying $d(x * y) \leq d(z)$ implies $\mu(d(x)) \geq \min \{\mu(d(y)), \mu(d(z))\}$.

Since $d(x *(x * y)) \leq d(y)$, it follows that $\mu(d(x)) \geq \min \{\mu(d(x * y)), \mu(d(y))\}$ Hence, $\mu$ is a derivation fuzzy ideal of $X$.

Now, we will apply the concept of derivation fuzzy ideal of $\mathbb{B C I}$-algebra to implicative ideals and prove many theorems. But before this we should define the derivation concept on implicative ideals of $\mathbb{B} \mathbb{C I}$-algebra $X$.

Let $(X, *, 0)$ be a $\mathbb{B} \mathbb{C I}$-algebra, $d: X \longrightarrow X$ be a self map. A non-empty subset $A$ of $\mathbb{B} \mathbb{C} \mathbb{I}$-algebra $X$ and $x, y, z \in X$ is called derivation implicative ideal of $\mathbb{B C I}$-algebra $X$ if it satisfies: $\left(\mathrm{D}_{1}\right) 0 \in A$ and $\left(\mathrm{DM}_{2}\right) d((x *(y * x)) * z) \in A$ and $d(z) \in A$ imply $d(x) \in A$, same applied for left and right derivation implicative ideals. 
Definition 3.10. Derivation $d: X \longrightarrow X$, fuzzy subset $\mu$ of $\mathbb{B} \mathbb{C I}$-algebra $X$ and a non-empty subset $A$ for all $x, y, z \in X$ is called; left derivation fuzzy implicative ideal of $X$ if it satisfies: $\left(\mathrm{LDFM}_{1}\right) \mu(0) \geq \mu(x)$ and $\left(\mathrm{LDFM}_{2}\right)$ $\mu(d(x)) \geq \min \{\mu(d((x *(y * x))) * z), \mu(d(z))\}$;right derivation fuzzy implicative ideal $\left(\mathrm{RDFM}_{1}\right) \mu(0) \geq \mu(x)$ and $\left(\mathrm{RDFM}_{2}\right) \mu(d(x)) \geq \min \{\mu((x *(y * x)) *$ $d(z)), \mu(d(z))\}$ and derivation fuzzy implicative ideal $\left(\mathrm{DFM}_{1}\right) \mu(0) \geq \mu(d(x))$ and $\left(\mathrm{DFM}_{2}\right) \mu(d(x)) \geq \min \{\mu(d((x *(y * x)) * z)), \mu(d(z))\}$

Example 3.11. Let $X=\{0, a, b\}$ be a $\mathbb{B C I}$-algebra with the following table: Define a map $d: X \longrightarrow X$ by

Table 1: Cayley table

\begin{tabular}{|c|c|c|c|}
\hline$*$ & 0 & $\mathrm{a}$ & $\mathrm{b}$ \\
\hline 0 & 0 & 0 & $\mathrm{~b}$ \\
\hline $\mathrm{a}$ & $\mathrm{a}$ & 0 & $\mathrm{~b}$ \\
\hline $\mathrm{b}$ & $\mathrm{b}$ & $\mathrm{b}$ & 0 \\
\hline
\end{tabular}

$$
d(x)= \begin{cases}0 & \text { if } x=0, a, \\ b & \text { if } x=b .\end{cases}
$$

Then by [16] $d$ is derivation of $X$. Now we define a fuzzy set for $t_{0}, t_{1} \in[0,1]$ and $t_{0} \geq t_{1}$ with $\mu: X \longrightarrow[0,1]$ by $\mu(0)=t_{0}, \mu(a)=\mu(b)=t_{1}$, and if we define derivation on the fuzzy set by $\mu: d(x) \longrightarrow[0,1]$ such that $\mu(d(0))=\mu(d(a))=$ $\mu(d(b))=t_{1}$ and $t_{1} \in[0,1]$. Then it is easily to check that $\mu$ is derivation fuzzy implicative ideal of $\mathbb{B} \mathbb{C} I$-algebra $X$.

Theorem 3.12. Any derivation fuzzy implicative ideals of $\mathbb{B} \mathbb{C I}$-algebra $X$ is derivation fuzzy $\mathbb{B} \mathbb{C I}$-ideals of $X$.

Proof. As given, $\mu$ is fuzzy derivation implicative ideals of $X$ then we have: $\mu(d(x)) \geq \min \{\mu(d((x *(y * x)) * z)), \mu(d(z))\}$ put $z=y$ and $y=x$ then we get

$$
\begin{aligned}
\mu(d(x)) & \geq \min \{\mu(d((x *(x * x)) * y)), \mu(d(y))\} \\
& =\min \{\mu(d((x * 0) * y)), \mu(d(y))\} \\
& =\min \{\mu(d(x * y)), \mu(d(y))\}
\end{aligned}
$$

and this is the fuzzy derivation $\mathbb{B C I}$ ideals of $\mathrm{X}$.

The converse of theorem (3.12) is not true always as showing in the next example. 
Example 3.13. $X=\{0, a, b\}$ with Cayley Table1, same derivation and fuzzy set defined in Example 3.11, if we define a derivation on the fuzzy set as: $\mu: d(x) \longrightarrow[0,1]$ with $\mu(d(0))=\mu(d(a))=t_{0}, \mu(d(b))=t_{1}$ easy calculation we find out that $\mu$ is derivation fuzzy ideal but it is not derivation fuzzy implicative ideal, if we take $x=b, y=a, z=0$ we get: $t_{1}=\mu(d(b)) \ngtr \mu(d(0))=t_{0}$.

Corollary 3.14. Every derivation fuzzy implicative ideal must be a derivation fuzzy sub-algebra of $X$.

Proof. proof follows from previous theorem.

Theorem 3.15. let $\mu$ be derivation fuzzy $\mathbb{B} \mathbb{C I}$-ideal of $X$, then $\mu$ is a regular derivation fuzzy implicative ideals if and only if it satisfies the following condition:

$$
\mu(d(x)) \geq \mu(d(x *(y * x)))
$$

Proof. " $\Rightarrow$ ": Suppose $\mu$ is regular derivation fuzzy implicative ideals and we will prove our condition, Take $z=0$ in $\left(\mathrm{DFM}_{2}\right)$, Hence we will have:

$$
\begin{aligned}
\mu(d(x)) & \geq \min \{\mu(d((x *(y * x)) * 0)), \mu(d(0))\} \\
& =\min \{\mu(d(x *(y * x))), \mu(d(0))\} \\
& =\mu(d(x *(y * x)))
\end{aligned}
$$

$" \Leftarrow "$ : we will prove that $\mu$ is regular derivation fuzzy implicative ideals. Since $\mu$ is derivation fuzzy $\mathbb{B} \mathbb{C I}$-ideal $\left(\mathrm{DFM}_{1}\right)$ satisfied and left to prove $\left(\mathrm{DFM}_{2}\right)$. from our condition then we can say that:

$$
\begin{aligned}
\mu(d(x)) & \geq \mu(d(x *(y * x))) \\
& \geq \min \{\mu(d((x *(y * x) * z))), \mu(d(z))\}
\end{aligned}
$$

And this is the derivation fuzzy implicative ideal.

Theorem 3.16. In implicative $\mathbb{B} \mathbb{C} \mathbb{K}$-algebra $X$, every derivation fuzzy $\mathbb{B C I}$-ideal of $X$ is a derivation fuzzy implicative ideal in $X$.

Proof. In implicative $\mathbb{B} \mathbb{C} K$-algebra, $x=x *(y * x)$ and since $\mu$ is derivation fuzzy $\mathbb{B C I}$-ideal $\left(\mathrm{DFM}_{1}\right)$ satisfied and left to prove $\left(\mathrm{DFM}_{2}\right)$ then we have:

$$
\mu(d(x)) \geq \min \{\mu(d(x * z)), \mu(d(z))\}
$$

substitute $x$ by $x=x *(y * x)$ in the right hand side and we will get: 


$$
\mu(d(x)) \geq \min \{\mu(d((x *(y * x)) * z)), \mu(d(z))\}
$$

and this is $\left(\mathrm{DFM}_{2}\right)$.

Theorem 3.17. The intersection of any set of derivation fuzzy implicative ideals on $\mathbb{B} \mathbb{C I}$-algebra $X$ is also a derivation fuzzy implicative ideal.

Proof. Let $\mu_{i}$ be a family of derivation fuzzy implicative ideals on $\mathbb{B C I}$ algebra $X$ and for any $x, y, z \in X$ we will show that the intersection of $\mu_{i}$ is a derivation fuzzy implicative ideal:

$\mathrm{DFM}_{1}:\left(\cap \mu_{i}\right)(0)=\inf \left(\mu_{i}\right)(0) \geq \inf \left(\mu_{i}\right)(x)=\left(\cap \mu_{i}\right)(x)$

$\mathrm{DFM}_{2}$ :

$$
\begin{aligned}
\left(\cap \mu_{i}\right)(d(x))=\inf \left(\mu_{i}\right)(d(x)) & \geq \inf \left(\min \left\{\mu_{i}(d((x *(y * x)) * z)), \mu_{i}(d(z))\right\}\right) \\
& =\min \left\{\inf \left(\mu_{i}(d((x *(y * x)) * z))\right), \inf \left(\mu_{i}(d(z))\right)\right\} \\
& =\min \left\{\cap\left(\mu_{i}\right)(d((x *(y * x)) * z)), \cap\left(\mu_{i}\right)(d(z))\right\}
\end{aligned}
$$

$\left(\cap \mu_{i}\right)(d(x)) \geq \min \left\{\cap\left(\mu_{i}\right)(d(x * y)), \cap\left(\mu_{i}\right)(d(y))\right\}$ and that's $\mathrm{FDM}_{2}$.

Same result applied for left and right derivation fuzzy implicative ideals of $\mathbb{B} \mathbb{C I}$-algebra $X$.

Theorem 3.18. The union of many derivations fuzzy implicative ideals on $\mathbb{B C I}$-algebra $X$ is also a derivation fuzzy implicative ideal.

Proof. let's say that $\mu_{1}$ and $\mu_{2}$ are two derivations fuzzy implicative ideals of $\mathbb{B C I}$-algebra $X$.

We will show that $\mu_{1} \cup \mu_{2}$ is also derivation fuzzy implicative ideals of $X$.

$\mathrm{DFM}_{1}:\left(\mu_{1} \cup \mu_{2}\right)(0)=\mu_{1}(0) \cup \mu_{2}(0) \geq \mu_{1}(x) \cup \mu_{2}(x)=\left(\mu_{1} \cup \mu_{2}\right)(x)$

$\mathrm{DFM}_{2}$ :

$$
\begin{aligned}
& \left(\mu_{1} \cup \mu_{2}\right)(d(x))=\mu_{1}(d(x)) \cup \mu_{2}(d(x)) \\
& \geq \min \left\{\mu_{1}(d((x *(y * x)) * z)), \mu_{1}(d(z))\right\} \cup \min \left\{\mu_{2}(d((x *(y * x)) * z)), \mu_{2}(d(z))\right\} \\
& =\min \left\{\mu_{1}(d((x *(y * x)) * z)) \cup \mu_{2}(d((x *(y * x)) * z)), \mu_{1}(d(z)) \cup \mu_{2}(d(z))\right\} \\
& =\min \left\{\left(\mu_{1} \cup \mu_{2}\right)(d((x *(y * x)) * z)),\left(\mu_{1} \cup \mu_{2}\right)(d(z))\right\}
\end{aligned}
$$

$\left(\mu_{1} \cup \mu_{2}\right)(d(x)) \geq \min \left\{\left(\mu_{1} \cup \mu_{2}\right)(d((x *(y * x)) * z)),\left(\mu_{1} \cup \mu_{2}\right)(d(z))\right\}$ and that's $\mathrm{DFM}_{2}$.

Same result applied for left and right derivation fuzzy implicative ideals of $\mathbb{B} \mathbb{C I}$-algebra $X$. 
Theorem 3.19. a fuzzy subset $\mu$ of $\mathbb{B} \mathbb{C I}$-algebra $X$ is derivation fuzzy implicative ideal of $X$ if and only if for every $t \in[0,1], \mu_{t}$ is either empty or derivation implicative ideal of $X$.

Proof. (i) " $\Rightarrow "$ : Assume $\mu$ is a derivation fuzzy implicative ideal of $X$,we have:

$\mu(0) \geq \mu(x)$ for any $x \in X$, Therefore $\mu(0) \geq \mu(x) \geq t$ then $x \in \mu_{t}$ and so, $0 \in \mu_{t}$ and this gives $\mu_{t} \neq \Phi$.

Now, let $d((x *(y * x)) * z) \in \mu_{t}$ and $d(z) \in \mu_{t}$

So, $\mu(d((x *(y * x)) * z)) \geq t$ and $\mu(d(z)) \geq t$

since $\mu$ is a derivation fuzzy implicative ideal we have:

$\mu(d(x)) \geq \min \{\mu(d((x *(y * x)) * z)), \mu(d(z))\} \geq t$

Hence, $\mu(d(x)) \geq t$ so, $d(x) \in \mu_{t}$ Thus, $\mu_{t}$ is derivation implicative ideal of $X$.

$" \Leftarrow "$ :we will show that $\mathrm{DFM}_{1}$ and $\mathrm{DFM}_{2}$ both are true.

Suppose $\mathrm{DFM}_{1}$ is not true, then there exist $x^{\prime} \in X$ such that $\mu(0) \leq \mu\left(x^{\prime}\right)$ and if we take: $t^{\prime}=\frac{\mu\left(x^{\prime}\right)+\mu(0)}{2}$ this gives us

$$
\mu(0) \leq t^{\prime}
$$

So, $0 \leq t^{\prime}<\mu\left(x^{\prime}\right) \leq 1$ then $\mu\left(x^{\prime}\right)>t^{\prime}, x^{\prime} \in \mu\left(t^{\prime}\right)$ and those $\mu\left(t^{\prime}\right) \neq \phi$

As $\mu\left(\overline{t^{\prime}}\right)$ is derivation implicative ideal of $X$, then we have $0 \in \mu\left(t^{\prime}\right)$ this gives $\mu(0) \geq t^{\prime}$ and this a contradiction with (1), so $\mathrm{DFM}_{1}$ is true.

Now, assume $\mathrm{DFM}_{2}$ is not true then there exist $x^{\prime}, y^{\prime}, z^{\prime} \in X$ such that:

$$
\mu\left(d\left(x^{\prime}\right)\right) \leq \min \left\{\mu\left(d\left(\left(x^{\prime} *\left(y^{\prime} * x^{\prime}\right)\right) * z^{\prime}\right)\right), \mu\left(d\left(z^{\prime}\right)\right)\right\}
$$

Put $t^{\prime}=\frac{\mu\left(d\left(x^{\prime}\right)\right)+\min \left\{\mu\left(d\left(\left(x^{\prime} *\left(y^{\prime} * x^{\prime}\right)\right) * z^{\prime}\right)\right), \mu\left(d\left(z^{\prime}\right)\right)\right\}}{2}$ this gives us:

$$
\mu\left(d\left(x^{\prime}\right) \leq t^{\prime}\right.
$$

So, $0 \leq t^{\prime}<\min \left\{\mu\left(d\left(\left(x^{\prime} *\left(y^{\prime} * x^{\prime}\right)\right) * z^{\prime}\right)\right), \mu\left(d\left(z^{\prime}\right)\right)\right\} \leq 1$

Thus, $\min \left\{\mu\left(d\left(\left(x^{\prime} *\left(y^{\prime} * x^{\prime}\right)\right) * z^{\prime}\right)\right), \mu\left(d\left(z^{\prime}\right)\right)\right\} \geq t^{\prime}$ that means: $\mu\left(d\left(\left(x^{\prime} *\left(y^{\prime} * x^{\prime}\right)\right) * z^{\prime}\right)\right) \geq t^{\prime}$ and $\mu\left(d\left(z^{\prime}\right)\right) \geq t^{\prime}$

Then, $d\left(\left(x^{\prime} *\left(y^{\prime} * x^{\prime}\right)\right) * z^{\prime}\right) \in \mu\left(t^{\prime}\right)$ and $d\left(z^{\prime}\right) \in \mu\left(t^{\prime}\right)$

Since $\mu\left(t^{\prime}\right)$ is derivation implicative ideal of $X$, it follows that $d\left(x^{\prime}\right) \in \mu\left(t^{\prime}\right)$ and we get that $\mu\left(d\left(x^{\prime}\right)\right)>t^{\prime}$ and this is a contradiction with (2) and so $\mathrm{DFM}_{2}$ is true and satisfied, therefore $\mu$ is derivation fuzzy implicative ideal of $X$. 
Proposition 3.20. If $I$ and $A$ are two ideals of $\mathbb{B} \mathbb{C I}$-algebra $X$ such that $I \subseteq A$, if $I$ is a derivation implicative ideal, then so is $A$.

Theorem 3.21. Let $\mu$ and $\nu$ be two fuzzy ideals of $\mathbb{B} \mathbb{C I}$-algebra $X$ such that: $\mu \leq \nu$ and $\mu(0)=\nu(0)$, if $\mu$ is derivation fuzzy implicative ideal so is $\nu$.

Proof. To prove $\nu$ is derivation fuzzy implicative ideal of $X$, it suffices to show that for any $t \in[0,1], \nu_{t}$ is either empty or derivation implicative ideal of $X$.

Suppose $\nu_{t}$ is non-empty so, we have to prove that $\nu_{t}$ is derivation implicative ideal. $\nu_{t} \neq \phi$ implies $\mu_{t} \neq \phi$ since $\mu_{t} \subseteq \nu_{t}$. moreover, $\mu_{t} \neq \phi$ then $x \in \mu_{t}$ and $\mu(x) \geq t$ so $\nu(x) \geq t$ and $x \in \nu_{t}$ hence, $\mu_{t} \subseteq \nu_{t}$.

Since, $\mu$ derivation fuzzy implicative ideal of $X$ By (3.19) $\mu_{t}$ is derivation implicative ideal of $X$ and by (3.20) and since $\mu_{t} \subseteq \nu_{t}$ so, $\nu_{t}$ is also derivation implicative ideal of $X$. Thus, $\nu$ is derivation fuzzy implicative ideal of $X$.

Theorem 3.22. Suppose that $\mu$ is a regular derivation fuzzy ideal of $X$ then that following are equivalent:

(i) $\mu$ is derivation fuzzy implicative ideal.

(ii) $\mu(d(x)) \geq \mu(d(x *(y * x)))$.

(iii) $\mu(d(x))=\mu(d(x *(y * x)))$

Proof. (i)" $\Rightarrow "\left(\right.$ ii): let $\mu$ be derivation fuzzy implicative ideal by $\left(\mathrm{DFM}_{2}\right)$ and let $z=0$ we have:

$$
\begin{aligned}
\mu(d(x)) & \geq \min \{\mu(d((x *(y * x)) * z)), \mu(d(z))\} \\
& =\min \{\mu(d((x *(y * x)) * 0)), \mu(d(0))\} \\
& =\mu(d(x *(y * x)))
\end{aligned}
$$

Therefore, $\mu(d(x)) \geq \mu(d(x *(y * x)))$

(ii)" $\Rightarrow$ "(iii): Since $d(x *(y * x)) \leq x *(y * x) \leq x$ then, $\mu(d(x)) \leq \mu(d(x *(y * x)))$

And since we have (ii) so we get (iii).

(iii)" $\Rightarrow "($ i): Suppose (iii) holds and since $\mu$ is derivation fuzzy ideal we have:

$\mu(d(x *(y * x))) \geq \min \{\mu(d((x *(y * x)) * z)), \mu(d(z))\}$ combining this equation with (iii) we will get $\left(\mathrm{DFM}_{2}\right), \mu(d(x)) \geq \min \{\mu(d((x *(y * x)) * z)), \mu(d(z))\}$. 
Proposition 3.23. In a $\mathbb{B} \mathbb{C} \mathbb{K}$-algebra $X$ the following hold for all $x, y, z \in$ $X:[15]$

(i) $((x * z) * z) *(y * z) \leq(x * y) * z$

(i) $(x * z) *(x *(x * z))=(x * z) * z$

(iii) $(x *(y *(y * x))) *(y *(x *(y *(y * x)))) \leq x * y$

Definition 3.24. A fuzzy subset $\mu$ in $X$ is called a derivation fuzzy commutative ideal of $X$ if it satisfies $\left(\mathrm{DF}_{1}\right)$ and

$\left(\mathrm{DFC}_{2}\right) \mu(d(x *(y *(y * x)))) \geq \min \{\mu(d((x * y) * z)), \mu(d(z))\}$ for all $x, y, z \in X$

Proposition 3.25. Let $\mu$ be a derivation fuzzy ideal of $X$. Then $\mu$ is derivation fuzzy commutative ideal if and only if it satisfies this condition for all $x, y \in X$ :

$$
\mu(d(x *(y *(y * x)))) \geq \mu(d(x * y))
$$

Proof. " $\Rightarrow "$ :Suppose $\mu$ is derivation fuzzy commutative ideal, let $z=0$ in $\left(\mathrm{DFC}_{2}\right)$ :

$$
\begin{aligned}
\mu(d(x *(y *(y * x)))) & \geq \min \{\mu(d((x * y) * 0)), \mu(d(0))\} \\
& =\min \{\mu(d(x * y)), \mu(d(0))\} \\
& =\mu(d(x * y))
\end{aligned}
$$

And this is (3).

$" \Leftarrow "$ : Suppose $(3)$ is given and since $\mu$ is derivation fuzzy ideal $\left(\mathrm{DFC}_{1}\right)$ satisfied and we have:

$\mu(d(x * y)) \geq \min \{\mu(d((x * y) * z)), \mu(d(z))\}$ combining this equation with $(3)$ we get:

$\mu(d(x *(y *(y * x)))) \geq \min \{\mu(d((x * y) * z)), \mu(d(z))\}$ and this is $\left(\mathrm{DFC}_{2}\right)$.

Hence, Proposition (3.25) can be improved as follows:

Theorem 3.26. A derivation fuzzy ideal $\mu$ of $X$ is derivation fuzzy commutative ideal if and only if it satisfies the identity:

$$
\mu(d(x * y))=\mu(d(x *(y *(y * x)))) \text { for all } x, y \in X
$$

Definition 3.27. A fuzzy subset $\mu$ in $X$ is called a derivation fuzzy positive implicative ideal of $X$ if it satisfies $\left(\mathrm{DF}_{1}\right)$ and $\left(\mathrm{DFPM}_{2}\right) \mu(d(x * z)) \geq \min \{\mu(d((x * y) * z)), \mu(d(y * z))\}$ for all $x, y, z \in X$. 
Proposition 3.28. A derivation fuzzy ideal $\mu$ of $X$ is derivation fuzzy positive implicative ideal if and only if it satisfies the condition for all $x, y \in X$ :

$$
\mu(d(x * y)) \geq \mu(d((x * y) * y))
$$

Proof. " $\Rightarrow "$ :Suppose $\mu$ is derivation fuzzy positive implicative ideal, take $z=y$ in $\left(\mathrm{DFPM}_{2}\right)$ :

$$
\begin{aligned}
\mu(d(x * y)) & \geq \min \{\mu(d((x * y) * y)), \mu(d(y * y))\} \\
& =\min \{\mu(d((x * y) * y)), \mu(d(0))\} \\
& =\mu(d((x * y) * y))
\end{aligned}
$$

Hence, this is (4)

$" \Leftarrow "$ : Suppose $(4)$ is given and $\mu$ is derivation fuzzy ideal so $\left(\mathrm{DFPM}_{1}\right)$ satisfied and left to prove $\left(\mathrm{DFPM}_{2}\right)$ :

$\mu(d(x)) \geq \min \{\mu(d(x * y)), \mu(d(y))\}$ Making use of (4) for both sides we get: $\mu(d(x * z)) \geq \min \{\mu(d((x * y) * z)), \mu(d(y * z))\}$ and this is $\left(\mathrm{DFPM}_{2}\right)$.

Proposition (3.28) can be improved as follows:

Theorem 3.29. A derivation fuzzy ideal $\mu$ of $X$ is derivation fuzzy positive implicative ideal if and only if it satisfies the identity:

$$
\mu(d(x * y))=\mu(d((x * y) * y)) \text { for all } x, y \in X
$$

In the next theorem, we describe the relation between the derivation of fuzzy implicative ideals and the derivation of fuzzy commutative and positive implicative ideals.

Theorem 3.30. A derivation fuzzy ideal $\mu$ of $X$ is derivation fuzzy implicative ideal if and only if $\mu$ is both derivation fuzzy commutative and derivation fuzzy positive implicative.

Proof. " $\Rightarrow$ :Suppose $\mu$ is derivation fuzzy implicative ideal of $X$ for all $x, y, z \in X$ we have by $(3.23(\mathrm{i}),(\mathrm{ii}),(3.22(\mathrm{iii}))$ and $(3.6)$ :

$$
\begin{aligned}
\min \{\mu(d((x * y) * z)), \mu(d((y * z)))\} & \leq \mu(d((x * z) * z)) \\
& =\mu(d((x * z) *(x *(x * z))) \\
& =\mu(d(x * z))
\end{aligned}
$$


Hence, $\mu(d(x * z)) \geq \min \{\mu(d((x * y) * z)), \mu(d((y * z)))\}$ and this is $\left(\mathrm{DFPM}_{2}\right)$. So, $\mu$ is derivation fuzzy positive implicative ideal of $X$.

Now, by (3.5), (3.22)(iii) and (3.23)(iii) we have:

$$
\begin{aligned}
\mu(d(x * y)) & \leq \mu(d((x *(y *(y * x))) *(y *(x *(y *(y * x))))) \\
& =\mu(d(x *(y *(y * x))))
\end{aligned}
$$

it follows that by $(3.25) \mu$ is derivation fuzzy commutative ideal of $X$.

$" \Leftarrow "$ :conversely, suppose that $\mu$ is both derivation fuzzy commutative and positive implicative ideals.

Since, $(y *(y * x)) *(y * x) \leq(x *(y * x))$ it follows form (3.5) we have:

$\mu(d(x *(y * x))) \leq \mu(d((y *(y * x)) *(y * x)))$ Using (3.29), we have:

$\mu(d((y *(y * x)) *(y * x)))=\mu(d(y *(y * x)))$ By combining the above two equations we get:

$$
\mu(d(x *(y * x))) \leq \mu(d(y *(y * x)))
$$

On the other hand, since $x * y \leq x *(y * x)$ by (3.5) we have:

$\mu(d(x *(y * x))) \leq \mu(d(x * y))$ since $\mu$ is derivation fuzzy commutative ideal of $X$ and by $(3.26)$ we have: $\mu(d(x * y))=\mu(d(x *(y *(y * x))))$

Hence, $\mu(d(x *(y * x))) \leq \mu(d(x *(y *(y * x))))$ Combining with equation (5) $\mu(d(x *(y * x))) \leq \min \{\mu(d(x *(y *(y * x)))), \mu(d(y *(y * x)))\} \leq \mu(d(x))$

Therefore, we get that $\mu(d(x)) \geq \mu(d(x *(y * x)))$ By (3.22) so $\mu$ is derivation fuzzy implicative ideal of $X$.

Theorem 3.31. A derivation fuzzy sub-algebra $\mu$ of $X$ is a derivation fuzzy implicative ideal if and only if it satisfies

(b) $d((x *(y * x)) * z) \leq d(u)$ implies $\mu(d(x)) \geq \min \{\mu(d(z)), \mu(d(u))\}$ for all $x, y, z, u \in X$.

Proof. Assume that $\mu$ is a derivation fuzzy implicative ideal of $X$ and let $x, y, z, u \in X$ be such that:

$d((x *(y * x) * z)) \leq d(u)$ since $\mu$ is a derivation fuzzy ideal of $X$, it follows from (3.6):

$\mu(d(x *(y * x))) \geq \min \{\mu(d(z)), \mu(d(u))\}$

Making use of the theorem $(3.22$,(iii)) we obtain $\mu(d(x))) \geq \min \{\mu(d(z)), \mu(d(u))\}$ Hence, $\mu$ satisfy (b).

Conversely, Suppose that $\mu$ satisfies (b), clearly $\mu$ satisfies $D F M_{1}$. Since, $d((x *(y * x)) *(x *(y * x)) * z)) \leq d(z)$, it follows from (b) that: 
$\mu(d(x)) \geq \min \{\mu(d((x *(y * x)) * z)), \mu(d(z))\}$ which shows that $\mu$ satisfies $D F M_{2}$, and so $\mu$ is derivation fuzzy implicative ideal of $X$.

\section{Conclusion}

At the present paper, we introduced the concept of derivation fuzzy implicative ideals and discussed new theorems and properties. Furthermore we presented the relationship between derivation fuzzy implicative ideal and derivation fuzzy commutative and positive implicative ideal through a theorem and various properties are proved.

\section{References}

[1] H. A. S. Abujabal and N. O. Alshehri, Some results on derivations of $\mathbb{B} \mathbb{C I}$-algebras, Journal of Natural Sciences and Mathematics, 46, (2006), 13-19.

[2] H. A. S. Abujabal and N. O. Alshehri, On left derivations of $\mathbb{B} \mathbb{C I}$-algebras, Soochow Journal of Mathematics, 33, No.3 (2007), 435-444.

[3] H. E. Bell and L. C. Kappe, Rings in which derivations satisfy certain algebraic conditions, Acta Mathematica Hungarica, 53, No.(3-4) (1989), 339-346, doi:10.1007/BF01953371.

[4] H. E. Bell and G. Mason, On derivations in near-rings and near-fields, North-Holland Mathematics Studies, 137, (1987), 31-35, doi:10.1016/S0304-0208(08)72283-7.

[5] K. Is'eki, On BCI-algebras, In Math. Seminar Notes (presently Kobe J. Math.), 8, (1980), 125-130.

[6] K. Is'eki and S. Tanaka, Ideal theory of $\mathbb{B} \mathbb{C K}$-algebras, Math.Japonica, 21, No.4 (1976), 351-366.

[7] K. Is'eki and S. Tanaka, An introduction to the theory of $\mathbb{B} \mathbb{C K}$-algebras, Math. Japonica, 23, No.1 (1978), 1-26.

[8] Y. B. Jun, Closed fuzzy ideals in $\mathbb{B C I}$-algebras, Math. Japonica, 38, No.1 (1993), 199-202.

[9] Y. B. Jun and E. H. Roh, Fuzzy commutative ideals of $\mathbb{B} \mathbb{C K}$-algebras, Fuzzy Sets and Systems, 64, (1994), 401-405, doi:10.1016/0165-0114(94)90163-5.

[10] Y. B. Jun and X. L. Xin, On derivations of $\mathbb{B} \mathbb{C I}$-algebras, Information Science, 159, No.3 (2004), 167-176, doi:10.1016/j.ins.2003.03.001.

[11] Y. B. Jun, S. M. Hong, J. Meng and X. L. Xin, Characterizations of fuzzy positive implicative ideals in $\mathbb{B} \mathbb{C K}$-algebras, Math. Japan, 40, (1994), 503-507.

[12] K. Kaya, Prime rings with $\alpha$ derivation, Hacettepe Bulletin of Natural Sciences and Engineering, 16, (1987), 63-71.

[13] A. Kordi and A. Moussavi, On fuzzy ideals of $\mathbb{B} \mathbb{C I}$-algebras, PU. MA, 18, No.(3/4) (2007), 301-310. 
[14] J. Meng and Y. B. Jun, $\mathbb{B} \mathbb{C K}$-algebras, Kyung Moon Sa Company, 4, (1994), 119-121.

[15] J. Meng,Y. B. Jun and H. S. Kim, Fuzzy implicative ideals of $\mathbb{B} \mathbb{C} K$-algebras, Fuzzy Sets and Systems, 89, (1997), 243-48, doi:10.1016/S0165-0114(96)00096-6.

[16] M. A. Ozturk,Y. Ceven and Y. B. Jun, Generalized Derivations of $\mathbb{B} \mathbb{C I}$-algebras, Honam Mathematical Journal, 31, No.4 (2009), 601-609, doi:10.5831/HMJ.2009.31.4.601.

[17] E. Posner, Derivations in prime rings, Proceedings of the American Mathematical Society, 8, No.6 (1957), 1093-1100, doi:10.1090/S0002-9939-1957-0095863-0.

[18] O. G. Xi, Fuzzy $\mathbb{B C K}$-algebras, Math. Japonica, 36, (1991), 935-942.

[19] L. A. Zadeh, Fuzzy Sets, Information and control, 8, No.3 (1965), 338-353, doi:10.1142/9789814261302_0001. 